05

\title{
Влияние аллотропического перехода в титане на его энергию межатомного взаимодействия
}

\author{
() В.И. Бетехтин, А.Г. Кадомцев, М.В. Нарыкова
}

Физико-технический институт им. А.Ф. Иоффе РАН, Санкт-Петербург E-mail: vladimir.betekhtin@mail.ioffe.ru, Maria.Narykova@mail.ioffe.ru

Поступило в Редакцию 20 февраля 2017 г.

Изучение и анализ зависимости долговечности от напряжения и температуры свидетельствуют об изменении энергии межатомного взаимодействия при аллотропическом переходе в титане. Для объемноцентрированного $\beta$-Ті (при температуре $T>1155 \mathrm{~K}$ ) эта энергия $\approx 485 \mathrm{~kJ} / \mathrm{mol}$, что хорошо совпадает с теплотой сублимации титана, а для гексагонального плотноупакованного $\alpha$-Ti $(T<1155 \mathrm{~K}) \approx 305 \mathrm{~kJ} / \mathrm{mol}$. Рассмотрены литературные данные, подтверждающие сделанные в данной работе выводы.

DOI: 10.21883/PJTF.2017.15.44870.16753

Титан и сплавы на его основе имеют в настоящее время широкую сферу практического применения. Поэтому проблема прогнозирования прочности и механической стабильности титана достаточно актуальна $[1-5]$.

Согласно кинетическому подходу к прочности [6], важное значение для решения этой проблемы имеет изучение зависимости долговечности (времени $\tau$ с момента нагружения до разрушения материала) от величины напряжения $(\sigma)$ и температуры $(T)$. Анализ этой зависимости позволяет получить информацию о физической природе процесса, ведущего к потере механической стабильности и разрушению. Для широкого круга материалов (металлы, композиты, полимеры и т.д.) в определенном интервале внешних $\sigma$ и $T$ эта зависимость имеет вид [6]

$$
\tau=\tau_{0} \exp \left(U_{0}-\frac{\gamma \sigma}{k T}\right)
$$

где $k$ - постоянная Больцмана; $\tau_{0}$ для всех твердых тел $\approx 10^{-13} \mathrm{~s}$, совпадая с периодом тепловых колебаний атомов; множитель при 
напряжении $\gamma$ является единственным структурно-чувствительным коэффициентом уравнения (1).

Особо важное значение при анализе уравнения (1) имеет величина начального (до приложения $\sigma$ ) энергетического барьера $U_{0}$. Установлено, что для всех изученных металлов величина $U_{0}$ хорошо совпадает со значениями теплот их сублимации, которая является количественной характеристикой межатомного взаимодействия. Для каждого металла значение $U_{0}$, как и теплота сублимации, оказалось постоянной величиной, не зависящей от его структурного состояния (размера зерен, содержания примесей и т.д.) [6].

Титан, как и ряд важных для современной техники металлов (Co, $\mathrm{Zr}, \mathrm{Fe})$, при изменении температуры испытывает аллотропические превращения, которые могут заметно влиять на его свойства, в частности на энергию связи между атомами.

Целью данной работы являлась оценка величины $U_{0}$ и, следовательно, энергии межатомного взаимодействия для титана с решеткой объемноцентрированного куба (ОЦК, Ті при $T>1155 \mathrm{~K})$ и гексагональной плотноупакованной решеткой (ГПУ, Ті при $T<1155 \mathrm{~K})$.

Исследования проводили в основном на техническом титане ВТ1-0 с содержанием примесей $\approx 0.28 \%$. Лишь для $\beta$-Ті был использован, помимо ВТ1-0, более чистый иодидный Ті (99.9\%).

Механические испытания проводились при одноосном растяжении в режиме ползучести в диапазоне изменения долговечности более шести порядков (от $10^{-1}$ до $3 \cdot 10^{5} \mathrm{~s}$; методика измерения малых $<1 \mathrm{~s}$ долговечностей приведена в [6]). Следует отметить, что необходимым условием корректной оценки энергии активации из феноменологических данных является тождественность тех элементов структуры, которые оказывают доминирующее влияние на долговечность в образцах, испытуемых при разных $\sigma$ и $T$ [7]. В данной работе таким структурным элементом являлся размер зерен. Для его стабилизации все образцы перед испытанием на долговечность отжигались при $T$, превышающей максимальную $T$ опыта. Так, образцы $\alpha$-Ті отжигались при $925 \mathrm{~K}$ в течение $40 \mathrm{~h}$. Образцы $\beta$-Ті отжигались при $1323 \mathrm{~K}$ в течение $1 \mathrm{~h}$. Отжиг и последующие испытания на долговечность проводились в условиях вакуума.

Рассмотрим сначала экспериментальные данные, полученные для $\alpha$-Ті. На рис. 1 показаны зависимости долговечности от напряжения и температуры. Видно, что в координатах $\lg \tau-\sigma$ (при разных $T=\mathrm{const}$ )

Письма в ЖТФ, 2017, том 43, вып. 15 

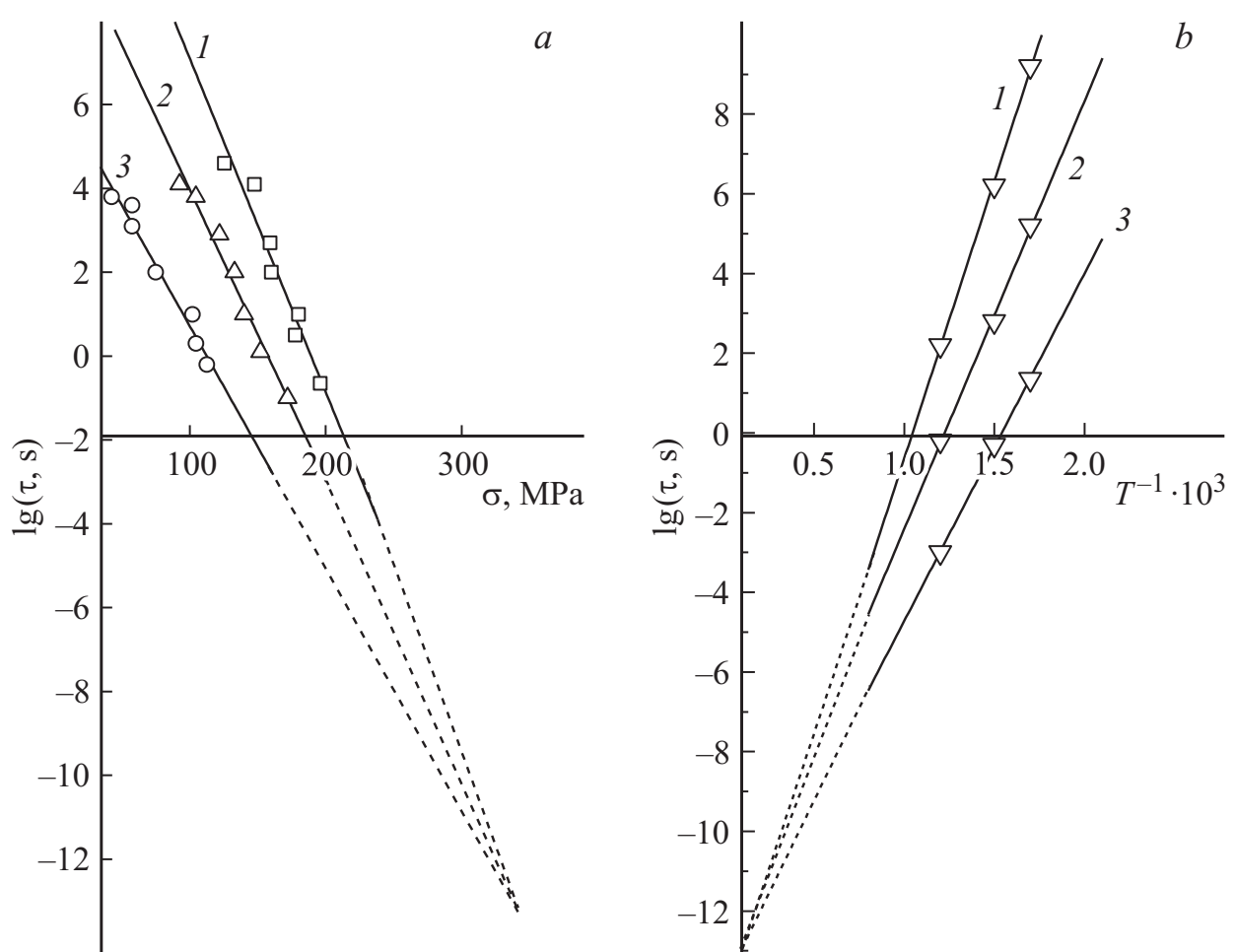

Рис. 1. Зависимость долговечности $\alpha$-Ті от: $a-$ напряжения $(1-T=573 \mathrm{~K}, 2-T=673 \mathrm{~K}, 3-T=823 \mathrm{~K})$; $b-$ обратной температуры $(1-\sigma=55 \mathrm{MPa}, 2-\sigma=125 \mathrm{MPa}, 3-\sigma=175 \mathrm{MPa})$. 


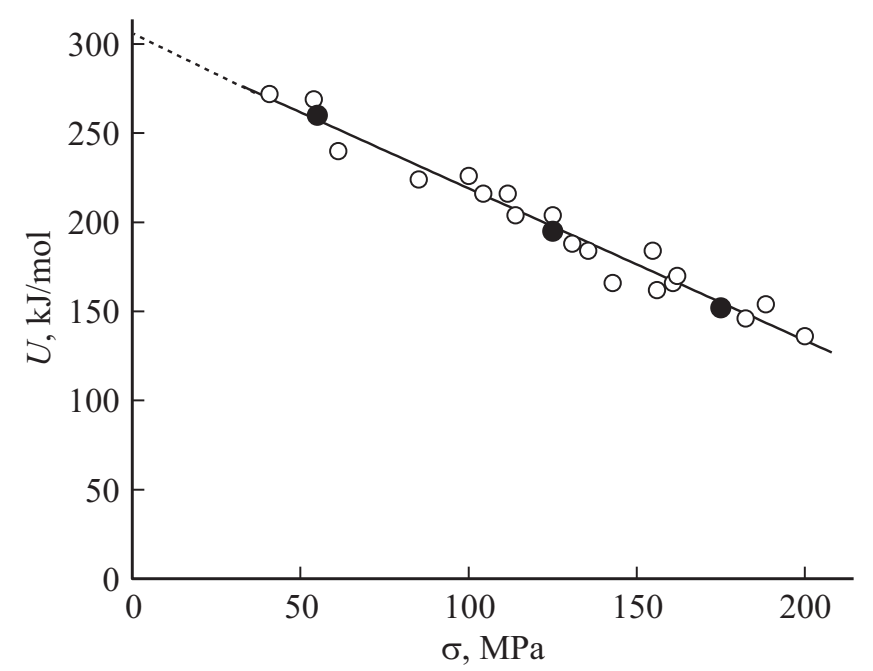

Рис. 2. Зависимость энергии активации от напряжения для $\alpha$-Тi.

и в координатах $\lg \tau-1 / T$ (построенных на основании данных рис. $1, a$ при разных $\sigma=\mathrm{const)} \mathrm{зависимости} \lg \tau$ от $\sigma$ и $1 / T$ представляют веер прямых линий, сходящихся при экстраполяции в полюсе. При этом, как и следовало из [6], полюс в координатах $\lg \tau-1 / T$ лежит на оси ординат, совпадая по своей величине с периодом тепловых колебаний атомов в твердом теле.

Последнее свидетельствует о выполнении формулы $\tau=\tau_{0} \exp \left(\frac{U(\sigma)}{k T}\right)$. Для определения зависимости $U$ от $\sigma$ из рис. $1, b$ вычисляются значения $U$ для трех $\sigma$ значений $\sigma$ и строится зависимость $U$ от $\sigma$. Видно (рис. 2), что эта зависимость линейна (черные точки на рис. 2). Убедившись в выполнении уравнения (1), зависимость $U$ от $\sigma$ можно построить на основании всех экспериментальных значений напряжения $\sigma$, приведенных на рис. $1, a$. Действительно, из уравнения (1) следует, что $U=k T \lg \frac{\tau}{\tau_{0}}$. Зная $\tau, T$, для каждой экспериментальной величины $\sigma$ можно (с учетом $\tau_{0}=10^{-13} \mathrm{~s}$ ) вычислить значение $U$. Полученная зависимость $U$ от $\sigma$ (светлые точки) также приведена на рис. 2. Линейный ход этой зависимости через большую совокупность точек позволяет путем небольшой экстраполяции на ось $\sigma=0$ доста- 


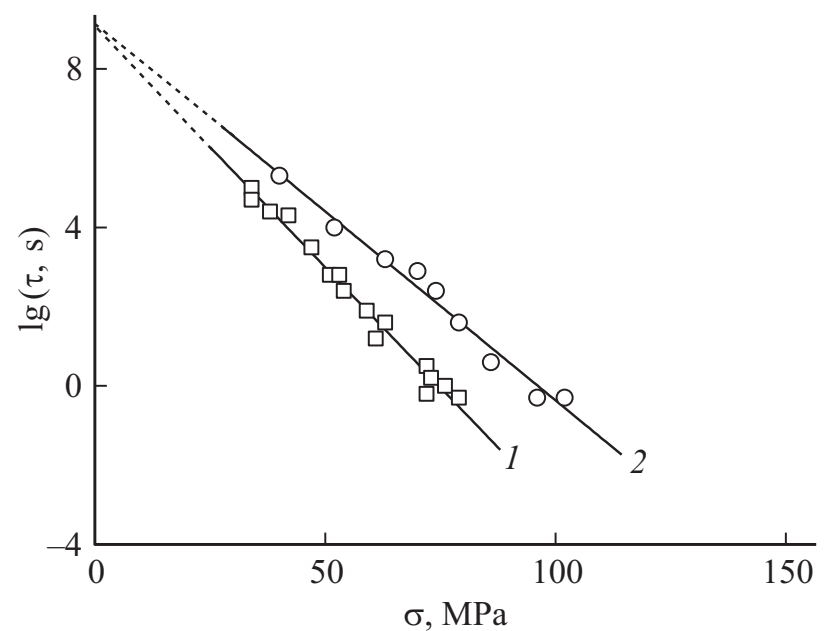

Рис. 3. Временнб́е зависимости прочности при постоянной температуре $T=1223 \mathrm{~K}: 1-\mathrm{Ti}(99.9 \%)$ иодидный, отжиг $1323 \mathrm{~K}, 1 \mathrm{~h} ; 2-\mathrm{Ti}(99.2 \%)$ ВТ1-0, отжиг $1323 \mathrm{~K}, 1 \mathrm{~h}$.

точно точно оценить величину $U_{0}$. Оказалось, что значение $U_{0}$ для $\alpha-\mathrm{Ti} \approx 305 \mathrm{~kJ} / \mathrm{mol}$.

Рассмотрим экспериментальные данные, полученные для $\beta$-модификации титана. На рис. 3 приведены зависимости долговечности от напряжения при постоянной температуре для разного по чистоте титана. Видно, что в координатах $\lg \tau-\sigma$ эти зависимости линейны, т.е. могут быть представлены уравнением

$$
\tau=A \exp (-\alpha \sigma)
$$

где коэффициент $\alpha$ связан с наклоном зависимости $\lg \tau-\sigma$, а коэффициент $A$ определяется при экстраполяции этой зависимости на ось ординат при $\sigma=0$.

Уравнение (2) является частным случаем более общей зависимости (1) [6]. Сопоставление уравнений (1) и (2) показывает, что $A=\tau_{0} \exp \left(\frac{U_{0}}{k T}\right)$ и, следовательно, $U_{0}=k T \lg \frac{A}{\tau_{0}}$. Таким образом, величину $U_{0}$ можно вычислить из анализа данных по временно́й зависимости, полученной при одной температуре (при обязательном условии

Письма в ЖТФ, 2017, том 43, вып. 15 
выполнения рассмотренных выше требований для корректной оценки энергии $\left.U_{0}\right)$. Из рис. 3 видно, что величина $A$ и, следовательно, $U_{0}$ для двух марок титана одинакова, численное значение $U_{0}$ оказалось $\approx 484 \mathrm{~kJ} / \mathrm{mol}$. Это значение хорошо совпадает с энергией сублимации титана $(480 \mathrm{~kJ} / \mathrm{mol})$, являющейся одной из важных энергетических характеристик межатомного взаимодействия. Как уже отмечалось, энергия сублимации не зависит от исходного состояния металла, в том числе от его чистоты. Равенство величин $A$ и, следовательно, $U_{0}$ для титана с разным содержанием примесей также свидетельствует в пользу „межатомной“ природы величины $U_{0}$.

Таким образом, если величина $U_{0}$ в уравнении (1) является одной из количественных характеристик межатомного взаимодействия, то полученные для титана данные свидетельствуют о существенном уменьшении энергии межатомных связей при аллотропическом переходе из ОЦК- в ГПУ-решетки.

Имеются также дополнительные аргументы, подтверждающие этот вывод. Известно, что межатомное взаимодействие связано с валентными электронами, которые для металлов коллективизированы. С этой точки зрения одной из важных количественных характеристик межатомного взаимодействия в металлах является энергия коллективного колебания валентных электронов (энергия плазмона $E_{p l}$ ), которая достаточно точно оценивается из экспериментальных данных по неупругому рассеянию быстрых электронов $[8,9]$ и определяется эффективной электронной концентрацией (долей электронов, участвующих в межатомном взаимодействии) [9-12]. В [12] показано, что для металлов первых трех периодов таблицы Менделеева имеется четкая корреляция между энергией плазмона и одной из наиболее надежных (также энергетической) характеристик межатомного взаимодействия - теплотой сублимации; для металлов с полиморфными превращениями эта корреляция усложняется. К сожалению, в литературе нет данных по $E_{p l}$ для двух решеток титана. Однако для Со, в котором имеет место аллотропический переход решетки ГПУ в гранецентрированную (ГЦК), установлено, что при переходе происходит увеличение $E_{p l}$ на 20\% [11]. Учитывая, что в титане полиморфный переход связан (в отличие от Со) с изменением координационного числа, можно ожидать, что изменение $E_{p l}$ при переходе ГПУ-ОЦК может быть еще больше.

В определенной степени межатомное взаимодействие характеризуется также плотностью состояний на уровне Ферми. Теория [13] и ее

Письма в ЖТФ, 2017, том 43, вып. 15 
экспериментальное подтверждение [14] показывают, что при переходе ГПУ-ОЦК в титане плотность состояний растет в 2 раза. Наконец, оценки, проведенные в [15], показали, что температура плавления (которая также зависит от межатомного взаимодействия) для $\beta$-Ті на $200 \mathrm{~K}$ выше, чем для $\alpha$-Тi.

Полученный в работе вывод об изменении энергии межатомного взаимодействия при полиморфном переходе в титане может оказаться полезным как при анализе природы механотермической стабильности высокопрочного наноструктурного Ті, приготовленного разными методами интенсивной пластической деформации [16-19], так и при выборе сплавов титана, содержащих ГПУ- и ОЦК-решетки. Следует, однако, отметить, что для сложных сплавов $\beta$-Ті, полученных за счет легирования большим количеством элементов разных периодов таблицы Менделеева $(\mathrm{Nb}, \mathrm{Mo}, \mathrm{Zr}$ и др.), для оценки энергии межатомного взаимодействия помимо эффективной электронной концентрации необходимо, вероятно, учитывать ряд других факторов (например, степень направленности и жесткости электронных конфигураций, экранирующее влияние внутренних электронов и т.д.).

Исследование выполнено за счет гранта Российского научного фонда (проект № 15-12-30010).

\section{Список литературы}

[1] Hyun C.Y., Kim H.K. // Rev. Mat. Sci. 2011. V. 28. N 1. P. 69-75.

[2] Kral P., Dvorak J., Sklenicka V. et al. // J. Mat. Sci. 2013. V. 48. N 13. P. 4789 4795.

[3] Wang Q., Yin Y. Sun Q. et al. // Mater. Res. 2014. V. 29. P. 569-577.

[4] Edalati K., Daio T., Arita M. et al. // Acta Mater. 2014. V. 68. P. 207-213.

[5] Banerjee D., Williams J.C. // Acta Mater. 2013. V. 61. P. 844-879.

[6] Регель В.Р., Слуикер А.И., Томашевский Э.Е. Кинетическая прочность твердых тел. М.: Наука, 1974. 560 с.

[7] Бетехтин В.И. // Проблемы прочности и пластичности твердых тел. К 100-летию акад. Н.Н. Давиденкова. Л.: Наука, 1979. С. 155-166.

[8] Fisher E.S., Dever D. // Acta Metal. 1970. V. 18. P. 205-269.

[9] Бакулин Е.А., Балабанова Л.А., Бредов М.М. // ФТТ. 1970. Т. 12. С. 72-76.

[10] Wehenkel C., Gauthe B. // Phys. Stat. Sol. B. 1974. V. 64. P. 515-521.

[11] Бакулин Е.А., Балабанова Л.А., Бредов М.М. // ФТТ. 1973. Т. 15. С. 2200 2202.

Письма в ЖТФ, 2017, том 43, вып. 15 
[12] Бакулин Е.А., Бетехтин В.И. // ФТТ. 1976. Т. 18. С. 3506-3508.

[13] Snow E.C., Waber J.T. // Acta Met. 1969. V. 17. P. 623-630.

[14] Ho I.C., Collings E.W. // Phys. Lett. F. 1969. V. 29. P. 206-208.

[15] Ardell A.J. // Acta Met. 1963. V. 11. N 6. P. 590-597.

[16] Dvorak J., Sklenicka V., Betekhtin V.I. et al. // Mat. Sci. Eng. A. 2013. V. 584. P. 103.

[17] Москаленко В.А., Бетехтин В.И., Кардашев Б.К. и др. // ФТТ. 2014. Т. 56. B. 8. C. $1539-1545$.

[18] Бетехтин В.И., Dvorak J., Кадомцев А.Г. и др. // Письма в ЖТФ. 2015. T. 41. B. 2. C. $58-63$.

[19] Бетехтин В.И., Колобов Ю.P., Sklenicka V. и др. // ЖТФ. 2015. Т. 85. В. 1. C. $66-71$. 